\title{
Cross-Functioning between the Extraneuronal Monoamine Transporter and Multidrug Resistance Protein 1 in the Uptake of Adrenaline and Export of 5-(Glutathion-S-yl)adrenaline in Rat Cardiomyocytes
}

\author{
Vera Marisa Costa,${ }^{*}{ }^{\dagger}$ Luísa Maria Ferreira, ${ }^{\ddagger}$ Paula Sério Branco ${ }^{\ddagger}{ }^{\ddagger}$ Félix Carvalho, ${ }^{\dagger}$ \\ Maria Lourdes Bastos, ${ }^{\dagger}$ Rui Albuquerque Carvalho, ${ }^{\S}$ Márcia Carvalho, ${ }^{\dagger, \|}$ and \\ Fernando Remião*,†

\begin{abstract}
REQUIMTE (Rede de Química e Tecnologia), Toxicology Department, Faculty of Pharmacy, University of Porto, Porto, Portugal, REQUIMTE, Departamento de Química, Faculdade de Ciências e Tecnologia, Universidade Nova de Lisboa, Caparica, Portugal, Neurosciences Center of Coimbra, Department of Faculty of Health Sciences, University Fernando Pessoa, Porto, Portugal
\end{abstract} \\ Biochemistry, Faculty of Sciences and Technology, University of Coimbra, Coimbra, Portugal, and CEBIMED,
}

Received July 15, 2008

\begin{abstract}
Isolated heart cells are highly susceptible to the toxicity of catecholamine oxidation products, namely, to catecholamine-glutathione adducts. Although cellular uptake and/or efflux of these products may constitute a crucial step, the knowledge about the involvement of transporters is still very scarce. This work aimed to contribute to the characterization of membrane transport mechanisms, namely, extraneuronal monoamine transporter (EMT), the multidrug resistant protein 1 (MRP1), and P-glycoprotein (P-gp) in freshly isolated cardiomyocytes from adult rats. These transporters may be accountable for uptake and/ or efflux of adrenaline and an adrenaline oxidation product, 5-(glutathion- $S$-yl)adrenaline, in cardiomyocyte suspensions. Our results showed that 5-(glutathion-S-yl)adrenaline efflux was mediated by MRP1. Additionally, we demonstrated that the adduct formation occurs within the cardiomyocytes, since EMT

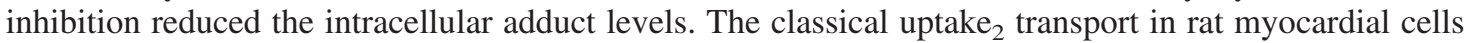
was inhibited by the typical EMT inhibitor, corticosterone, and surprisingly was also inhibited by low concentrations of another drug, a well-known P-gp inhibitor, GF120918. The P-gp activity was absent in the cells since P-gp-mediated efflux of quinidine was not blocked by GF120918. In conclusion, this work showed that freshly isolated cardiomyocytes from adult rats constitute a good model for the study of catecholamines and catecholamines metabolites membrane transport. The cardiomyocytes maintain EMT and MRP1 fully active, and these transporters contribute to the formation and efflux of 5-(glutathion$S$-yl)adrenaline. In the present experimental conditions, P-gp activity is absent in the isolated cardiomyocytes.
\end{abstract}

\section{Introduction}

There is increasing evidence that catecholamine oxidation may result in toxicity, namely, cardiotoxicity $(1-8)$. In cardiomyocytes, the oxidation process of adrenaline (ADR $)^{1}$ initially involves its conversion to $o$-quinone through $o$-semiquinone intermediary (5). The $o$-quinone can react with glutathione (GSH) to form GSH conjugates or be attacked by other nucleophilic groups in the cells. We reported previously, for the first time, the detection of 5-(glutathion- $S$-yl)adrenaline in cardiomyocytes, even at ADR concentrations as low as $25 \mu \mathrm{M}$ (5). Moreover, we observed that this GSH adduct was found in the incubation medium, leading to the hypothesis that a transporter-mediated efflux could be present (5). Certain GSH

* To whom correspondence should be addressed. Tel: 00351-222078979. Fax: 00351-222003977. E-mail: veramcosta@ff.up.pt (V.M.C.) or remiao@ ff.up.pt (F.R.).

University of Porto.

Universidade Nova de Lisboa.

$\S$ University of Coimbra.

"University Fernando Pessoa.

${ }^{1}$ Abbreviations: ABC, ATP-binding cassette; ADR, adrenaline; CORT, corticosterone; EMT, extraneuronal monoamine transporter; GSH, glutathione; LDH, lactate dehydrogenase; MRP1, multidrug resistance protein 1; P-gp, P-glycoprotein; PMAT, plasma membrane monoamine transporter conjugates are reported to be toxic in many organs $(9,10)$. In fact, they seem to be involved in the pathogenesis of Parkinson's disease $(11,12)$ and in the toxicity of illicit drugs, like 3,4methylenedioxymethamphetamine (MDMA) (13-15) or methamphetamine (16).

Cardiomyocytes are able, as other cells, to use efflux transporters to prevent the accumulation of compounds, leading to a decrease in their intracellular concentration (17). In 1976, a membrane-bound transporter, responsible for the efflux of anticancer drugs in cancer cells, was found and termed permeability glycoprotein (P-glycoprotein, P-gp) (18). P-gp conferred cancer cells the phenotype of "multidrug resistance". The multidrug resistance cell phenotype classically refers to tumors that are refractory to chemically unrelated cytotoxic agents, due to active outward transport. The P-gp expression does not fully account for all spectra of multidrug resistance. There are several ATP-binding cassette $(\mathrm{ABC})$ transporters involved in multidrug resistance, including P-gp [MDR1/ABCB1 (18)], the multidrug resistance protein 1 [MRP1/ABCC1 (19)], and the breast cancer resistance protein [ABCG2/BCRP/MXR/ABCP (20)]. These transporters are located in the plasma membrane and extrude a variety of drugs by using ATP (21-25). 
GSH-conjugated metabolites, once formed intracellularly, are usually exported to be eliminated from the body or act on their cellular targets. The efflux of GSH metabolites has already been reported as a consequence of a phase II metabolism (26) and is often mediated by the MRP1 transporter $(23,24)$. In addition to drugs and GSH conjugates, MRP1 exports GSH and glutathione disulfide (GSSG) and might thus have a role in cellular responses to oxidative stress (26). To meet the criteria for active transport, MRP1 transport should be ATP dependent, saturable, temperature dependent, osmotically sensitive, and inhibited by certain small molecules (e.g., MK-571) or MRP1specific monoclonal antibodies (26).

In this work, the ADR oxidation process was studied in isolated cardiomyocytes, by following the membrane transport of ADR and of its oxidation products. Catecholamines are translocated across plasma membranes by transporters that belong to two large families with mainly neuronal or extraneuronal locations (27). A low-affinity, high-capacity uptake for noradrenaline in the isolated perfused rat heart was found by Iversen in 1965 (28), while in 1967, Malmfors demonstrated by fluorescence microscopy that the myocytes are responsible for this uptake process (29). Extraneuronal uptake of catecholamines is mediated by organic cation transporters (OCTs), including OCT3, the classic corticosterone (CORT)-sensitive extraneuronal monoamine transporter (EMT), traditionally known as uptake ${ }_{2}$. Other transporters, like OCT1 and OCT2, are involved in catecholamine uptake, mainly in liver or kidney (30). The mRNA of plasma membrane monoamine transporter (PMAT) has been described in the heart, and PMAT is also able to transport organic cations $(31,32)$.

The present work aimed to characterize the functional transporters in freshly isolated cardiomyocytes, namely, those involved in the extraneuronal uptake of ADR and also the ABC transporters, MRP1 and P-gp. In addition, the transporters engaged in the formation and efflux of 5-(glutathion- $S$-yl)adrenaline were investigated in these cells.

\section{Materials and Methods}

Animals. Adult male Sprague-Dawley rats (Charles River Laboratories, Barcelona, Spain) weighing 250-350 g were used. The animals were housed in cages with a temperature- and humidity-controlled environment and a $12 \mathrm{~h}$ light-dark cycle. Food and water were provided ad libitum. Animal experiments were licensed by the Portuguese General Directory of Veterinary Medicine. Housing and experimental treatment of the animals were in accordance with the Guide for the Care and Use of Laboratory Animals from the Institute for Laboratory Animal Research (ILAR 1996). The experiments complied with current Portuguese laws.

Chemicals. All reagents used in this study were of analytical grade. Collagenase type II was obtained from Worthington (Lakewood, NJ). Collagenase (type IA), $N$-(2-hydroxyethyl) piperazine$N$-(2-ethanesulfonic acid) (HEPES), ADR, verapamil, cyclosporin, and CORT were obtained from Sigma-Aldrich (St. Louis, MO). The MRP1 inhibitor, MK-571 \{(E)-3-[[[3-[2-(7-chloro-2-quinolinyl)ethenyl]phenyl]-[[3-dimethylamino)-3-oxopropyl]thio]methyl]thio]propanoic acid\} was obtained from Calbiochem (San Diego, CA). GF120918 \{9,10-dihydro-5-methoxy-9-oxo-N-[4-[2-(1,2,3,4tetrahydro-6,7-dimethoxy-2-isoquinolinyl)ethyl]phenyl]-4-acridinecarboxamide\} was a generous gift from GlaxoSmithKline (Hertfordshire, United Kingdom). Dimethyl sulfoxide (DMSO) and perchloric acid $\left(\mathrm{HClO}_{4}\right)$ were purchased to Merck (Darmstadt, Germany).

Isolation of Calcium-Tolerant Cardiomyocytes from Adult Rat. Calcium-tolerant cardiomyocytes were isolated by Langendorff retro perfusion of adult rat heart, as previously described (5). At the beginning of the experiments, cell viability was always greater than $60 \%$, evaluated by the lactate dehydrogenase (LDH) leakage assay and by microscopic evaluation of the cardiomyocytes morphology. Incubations were performed in a water bath at $37^{\circ} \mathrm{C}$, using a density of $2.5 \times 10^{5}$ cells $/ \mathrm{mL}$ in modified Krebs-Henseleit buffer supplemented with $1 \mathrm{mM} \mathrm{CaCl}_{2}$ (pH 7.4) and saturated, every hour, with an airstream of carbogen.

After a preincubation of $30 \mathrm{~min}$, the cells were then exposed to the different compounds, namely, ADR; CORT, an EMT inhibitor; cyclosporin, verapamil, and GF120918, P-gp inhibitors; quinidine, an OCT1, OCT2, and PMAT inhibitor and P-gp substrate; and MK571, a MRP1 inhibitor, through a $3 \mathrm{~h}$ time study.

Sample Treatment. At the end of the $3 \mathrm{~h}$ incubation, cell samples were obtained after centrifugation of cardiomyocytes suspensions at $18 g$ for $2 \mathrm{~min}$. The pellet was washed twice with 1 $\mathrm{mL}$ of modified Krebs-Henseleit buffer supplemented with $1 \mathrm{mM}$ $\mathrm{CaCl}_{2}$, centrifuged at $18 \mathrm{~g}$ for $2 \mathrm{~min}$, and finally treated according to the different determinations. Washing solutions obtained after centrifugation were discarded.

HPLC/EC and HPLC/UV Analysis. The quantification of ADR and of the adrenaline-GSH adduct in the cardiomyocytes was performed using a HPLC instrument equipped with an electrochemical detector (EC), through a previously validated method (33). Quinidine determination was performed using the HPLC instrument coupled to UV detector, at 230 and $251 \mathrm{~nm}$, according to the work performed by Varma and co-workers (34).

Study of the Role of EMT in the Cardiomyocytes. To test whether EMT was involved in ADR uptake into cardiomyocytes, an inhibitor of uptake $e_{2}$, CORT, was tested at a final concentration of $100 \mu \mathrm{M}$ (35). Cardiomyocytes were preincubated with CORT for $30 \mathrm{~min}$, followed by an exposure to two different concentrations of ADR, 10 (concentration of ADR for which the GSH-adduct is not detectable in the cells) and $500 \mu \mathrm{M}$, for $3 \mathrm{~h}$.

Also to appraise whether adrenaline-GSH adduct could be a substrate of EMT, the cardiomyocytes were exposed to $14 \mu \mathrm{M}$ preformed 5-(glutathion- $S$-yl)adrenaline with and without CORT $(100 \mu \mathrm{M})$. The synthesis and isolation of the ADR adduct with GSH followed previously published methods (5).

MRP1 and 5-(Glutathion-S-yl)adrenaline. After the preincubation period, the cardiomyocytes were incubated with $500 \mu \mathrm{M}$ ADR for $3 \mathrm{~h}$, in either the presence or the absence of MK-571, a leukotriene $\mathrm{D}_{4}$ receptor antagonist, and a MRP1 inhibitor $(10 \mu \mathrm{M})$ (17). This experiment aimed to evaluate the involvement of MRP1 in the 5-(glutathion- $S$-yl)adrenaline efflux from the cardiomyocytes.

Measurement of Total GSH (GSHt) and GSSG Levels. In cardiomyocytes, the GSHt and GSSG were determined to appraise the interference of the MRP1 inhibitor in their values. The cardiomyocytes were subjected to the sample treatment previously described, acidified with $5 \% \mathrm{HClO}_{4}$, and centrifuged, and the supernatant obtained was used for the measurements by the DTNBGSSG redutase recycling assay (36).

Evaluation of P-gp Efflux. To evaluate the P-gp activity in cardiomyocytes, a P-gp substrate, quinidine $(100 \mu \mathrm{M})$, was used. Cardiomyocytes were preincubated for $30 \mathrm{~min}$ with quinidine, after which they were exposed to GF120918 $(10 \mu \mathrm{M})$. Quinidine levels in the cardiomyocytes were evaluated by HPLC/UV.

Evaluation of ADR and 5-(Glutathion-S-yl)adrenaline Levels in Cardiomyocytes Exposed to P-gp and OCT Inhibitors. P-gp inhibitors GF120918, cyclosporin, or verapamil were used to evaluate their interference in either 5-(glutathion- $S$ yl)adrenaline formation or efflux $(17,37,38)$. Each experiment entailed a control group (no treatment), an ADR group, an ADR plus P-gp inhibitor group, and a P-gp inhibitor group. When using GF120918 or cyclosporin, a group with only the vehicle (DMSO) was also tested. Cardiomyocytes were incubated with $500 \mu \mathrm{M}$ ADR in the presence and absence of P-gp inhibitors: $0.1,1$, and $10 \mu \mathrm{M}$ GF120918, $10 \mu \mathrm{M}$ verapamil, or 3 and $12 \mu \mathrm{M}$ cyclosporin. Studies with $20 \mu \mathrm{M}$ GF120918 were also performed.

Quinidine is a P-gp substrate and also an OCT1, OCT2, and PMAT inhibitor (31). It was used to evaluate the influence of transporters other than EMT in ADR transport in freshly isolated cardiomyocytes. Cardiomyocytes were preincubated with quinidine 

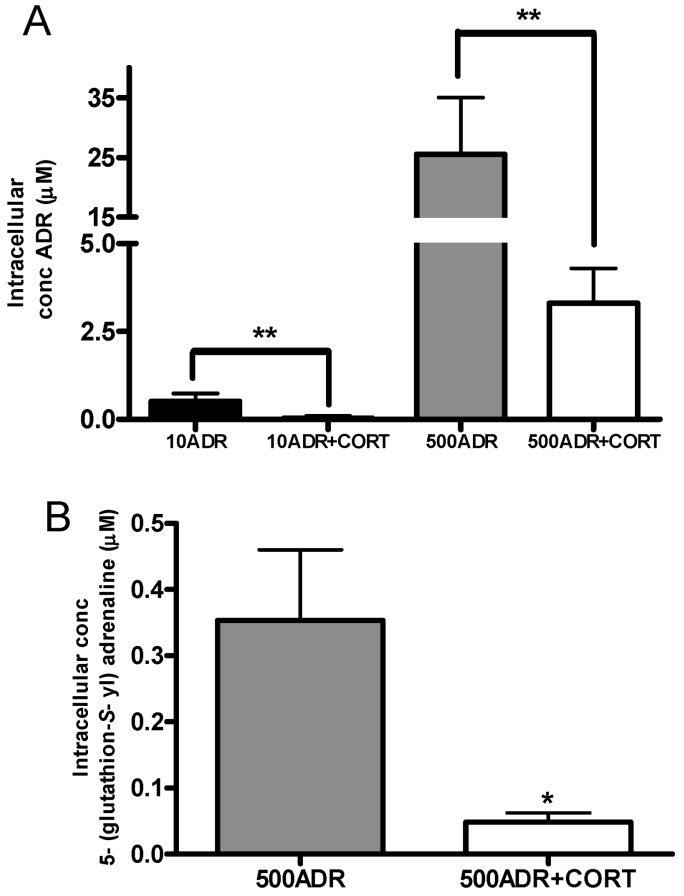

Figure 1. (A) Intracellular levels of adrenaline (ADR) in cardiomyocytes incubated for $3 \mathrm{~h}$ with $10 \mu \mathrm{M}$ ADR, $10 \mu \mathrm{M}$ ADR with CORT at $100 \mu \mathrm{M}, 500 \mu \mathrm{M}$ ADR, and $500 \mu \mathrm{M}$ ADR with CORT. Results are presented as means $(\mu \mathrm{M}) \pm \mathrm{SD}$ from six different experiments. Statistical comparisons were made using Kruskal-Wallis test followed by the Student-Newman-Keuls post hoc test $(* * p<0.01$ vs the respective group with no CORT). (B) Intracellular levels of 5-(glutathion- $S$-yl)adrenaline formed in cardiomyocytes exposed to $500 \mu \mathrm{M}$ ADR and $500 \mu \mathrm{M}$ ADR with CORT at $100 \mu \mathrm{M}$ for $3 \mathrm{~h}$. Statistical comparisons were made using the Mann-Whitney Ram Sum test $\left({ }^{*} p\right.$ $<0.05$ vs $500 \mu \mathrm{M}$ ADR).

for $30 \mathrm{~min}$, followed by incubation with $500 \mu \mathrm{M}$ ADR. The cellular content of ADR and 5-(glutathion-S-yl)adrenaline was monitored by HPLC.

LDH Leakage Assay. LDH leakage assay was performed directly in the cardiomyocyte suspensions to evaluate the level of cell injury at time $0 \mathrm{~h}$ (immediately after addition of the compounds) and after the $3 \mathrm{~h}$ incubation time in all treatments, as previously described (5).

Statistical Analysis. Results are given as means \pm the standard deviation (SD) from 4 to 6 independent experiments with suspensions of cardiomyocytes obtained from 4 to 6 different rats. Nonparametric tests were used. Statistical comparisons between groups were performed with a Kruskal-Wallis test (one-way ANOVA on Ranks) followed by the Student-Newman-Keuls post hoc test, once a significant $p$ had been obtained.

When only two treatment groups were compared, the MannWhitney Rank Sum test was used. Details of the statistical analysis are described in each figure legend. Significance was accepted at $p$ values of $<0.05$.

\section{Results}

Inhibition of EMT Leads to the Decrease in Intracellular Content of ADR and 5-(Glutathion-S-yl)adrenaline. The specific accumulation of ADR in cardiomyocytes, when incubated with 10 or $500 \mu \mathrm{M}$ ADR, was reduced by the EMT inhibitor, CORT $(100 \mu \mathrm{M})$. After incubation for $3 \mathrm{~h}$, a significantly higher concentration of intracellular ADR content was found in $10 \mu \mathrm{M}$ ADR group $(0.51 \pm 0.23 \mu \mathrm{M}$ ADR $)$ when compared with the $10 \mu \mathrm{M}$ ADR plus CORT $(0.03 \pm 0.06 \mu \mathrm{M}$ ADR) (Figure 1A). The same tendency was found in the 500 $\mu \mathrm{M}$ ADR group. The intracellular ADR concentration was 25.5 \pm 9.5 and $3.31 \pm 0.99 \mu \mathrm{M}$ in cells incubated with $500 \mu \mathrm{M}$ ADR and $500 \mu \mathrm{M}$ ADR plus CORT, respectively (Figure 1A).

As a product of ADR oxidation, 5-(glutathion- $S$-yl)adrenaline levels were shown to be dependent on the intracellular ADR concentration (5), as this work further demonstrates. In fact, with ADR incubation, 5-(glutathion- $S$-yl)adrenaline was evaluated in the cardiomyocytes, and its concentration was significantly reduced in the $500 \mu \mathrm{M}$ ADR plus CORT [0.049 \pm 0.014 $\mu \mathrm{M} 5$-(glutathion- $S$-yl)adrenaline], when compared with $500 \mu \mathrm{M}$ ADR [0.354 $\pm 0.106 \mu \mathrm{M} 5$-(glutathion- $S$-yl)adrenaline] (Figure 1B).

DMSO (2\%) was required to prepare CORT solutions, and it did not diminish the ADR transport into the cardiomyocytes or the 5-(glutathion-S-yl)adrenaline synthesis within the cells (data not shown). No interfering peaks in the chromatogram were found in control group, CORT, or vehicle group (data not shown).

To evaluate the possible involvement of EMT in the transport of preformed 5-(glutathion-S-yl)adrenaline into the cells, we incubated the cardiomyocytes with preformed 5-(glutathion- $S$ yl)adrenaline $(14 \mu \mathrm{M})$ with or without CORT. No differences were observed between the two groups (data not shown).

MRP1 System Is Involved in the Efflux of 5-(Glutathion-S-yl)adrenaline and GSSG. As MRP1 has been associated with the efflux of GSH and GSH conjugates $(17,26)$, its contribution toward the efflux of 5-(glutathion-S-yl)adrenaline was investigated in freshly isolated cardiomyocytes. Incubation of these cardiomyocytes with MK-571, a MRP1 inhibitor, led to the intracellular increase in 5-(glutathion- $S$-yl)adrenaline at $3 \mathrm{~h}$ in the ADR plus MK-571 group [0.462 $\pm 0.109 \mu \mathrm{M}$ 5-(glutathion- $S$-yl)adrenaline] when compared with only ADRtreated cells $[0.285 \pm 0.110 \mu \mathrm{M}$ 5-(glutathion- $S$-yl)adrenaline $]$ (Figure 2A).

Neither MK-571 nor its vehicle (DMSO 0.2\%) affected ADR levels within the cardiomyocytes (data not shown). The cellular GSHt (GSH + GSSG) and GSSG content was measured under MRP1 inhibition by MK-571. The cardiomyocytes in the ADR group had no change in GSSG levels, when compared to control, while GSSG significantly increased in the ADR plus MK-571 group when compared to control, ADR, or MK-571 groups (Figure 2B). The ADR group had a significant decrease in GSHt (Figure 2C). When MK-571 was also added to ADR, the GSHt in the cardiomyocytes increased when compared to the group with only ADR (Figure 2C). Neither MK-571 nor the vehicle (DMSO 0.2\%) affected GSSG or GSHt levels of cardiomyocytes (data not shown).

P-gp Inhibitor Affected EMT in Freshly Isolated Cardiomyocytes. To investigate other efflux transporters besides MRP1 or EMT, P-gp activity was evaluated in the isolated cells. The P-gp substrate, quinidine at $100 \mu \mathrm{M}$, and a P-gp inhibitor, GF120918 $(10 \mu \mathrm{M})$, were used. Although quinidine was found in the cardiomyocytes, no increase in its concentration was observed when the P-gp inhibitor was added (data not shown).

In independent experiments and to investigate the interference of P-gp inhibitors in the 5-(glutathion- $S$-yl)adrenaline formation or efflux, the cardiomyocytes were incubated with ADR (500 $\mu \mathrm{M}$ ) with or without P-gp inhibitors or substrates, namely, cyclosporin, verapamil, quinidine, and GF120918.

GSH adduct concentration in cardiomyocytes incubated simultaneously with $500 \mu \mathrm{M}$ ADR and $10 \mu \mathrm{M}$ GF120918 decreased significantly $[0.081 \pm 0.028 \mu \mathrm{M}$ 5-(glutathion- $S$ yl)adrenaline] when compared to ADR $[0.373 \pm 0.112 \mu \mathrm{M}$ 5-(glutathion- $S$-yl)adrenaline]. Additional incubation with MK$571(500 \mu \mathrm{M}$ ADR plus $10 \mu \mathrm{M}$ GF120918 and $10 \mu \mathrm{M}$ MK- 

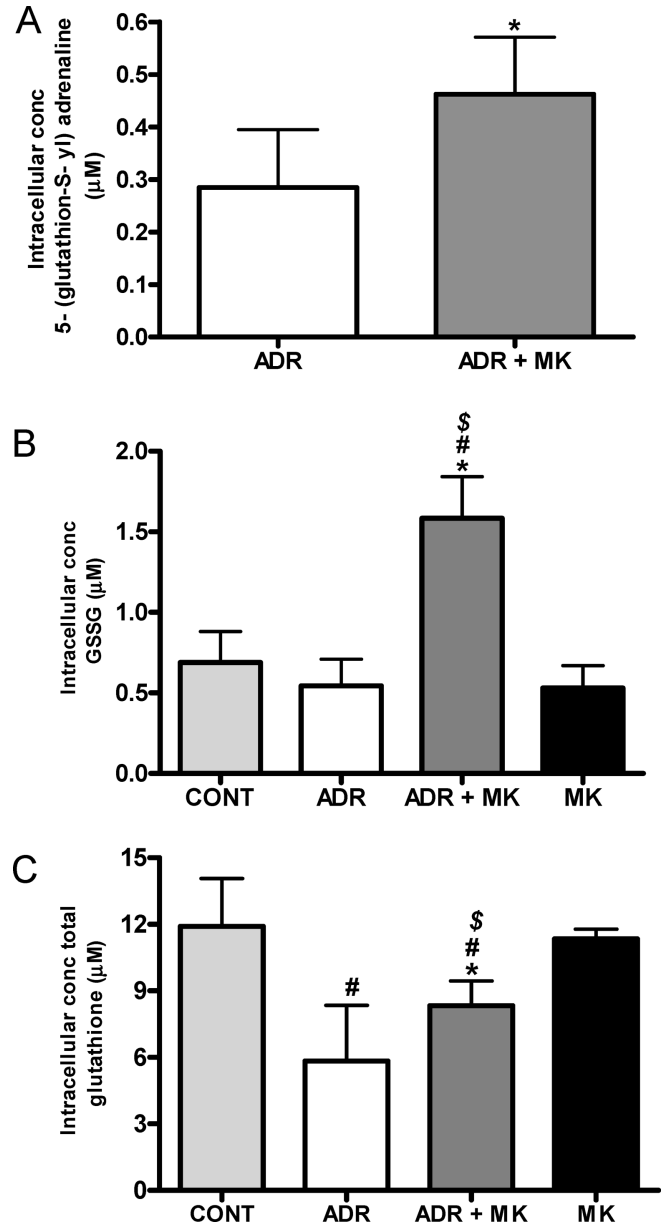

Figure 2. (A) Intracellular levels of 5-(glutathion- $S$-yl)adrenaline in calcium-tolerant rat cardiomyocytes exposed to $500 \mu \mathrm{M}$ ADR and 500 $\mu \mathrm{M}$ ADR plus MK-571 (MK) at $10 \mu \mathrm{M}$ for $3 \mathrm{~h}$. Results are presented as means \pm SD from six different experiments. Statistical comparisons were made using the Mann-Whitney Ram Sum test $\left({ }^{*} p<0.05\right.$, ADR vs ADR plus MK-571 group). (B) Intracellular GSSG levels in calciumtolerant rat cardiomyocytes in control group and groups exposed to $500 \mu \mathrm{M}$ ADR and $500 \mu \mathrm{M}$ ADR plus MK-571 for $3 \mathrm{~h}$ or MK-571 alone. Results are presented as means \pm SD from four different experiments. Statistical comparisons were made using Kruskal-Wallis test, followed by the Student-Newman-Keuls post hoc test $\left(^{*} p<\right.$ 0.05 vs $500 \mu \mathrm{M}$ ADR; ${ }^{*} p<0.05$ vs control; and ${ }^{\$} p<0.05$ vs MK571). (C) GSHt levels in calcium-tolerant rat cardiomyocytes in control group and groups exposed to $500 \mu \mathrm{M}$ ADR, $500 \mu \mathrm{M}$ ADR plus MK571 , and MK-571 at $3 \mathrm{~h}$. Results are presented as means \pm SD from four different experiments. Statistical comparisons were made using Kruskal-Wallis test, followed by the Student-Newman-Keuls post hoc test $\left(* p<0.05\right.$ vs $500 \mu \mathrm{M}$ ADR; ${ }^{*} p<0.05$ vs control; and ${ }^{\$} p<$ 0.05 vs MK-571).

571) resulted in a slight increase $[0.091 \pm 0.048 \mu \mathrm{M} 5$-(glutathion-S-yl)adrenaline] (Figure 3A).

After observing the decrease of 5-(glutathion-S-yl)adrenaline in cardiomyocytes when incubated with GF120918, we decided to study the possible inhibition of EMT by GF120918. We measured the ADR concentration in cardiomyocytes when using $10 \mu \mathrm{M}$ GF120918. The concentration of intracellular ADR was greatly decreased in the ADR plus GF120918 group when compared to ADR group (Figure 3B). This showed that GF120918 severely decreased ADR levels within the cardiomyocytes, which was reflected in the intracellular formation of 5-(glutathion- $S$-yl)adrenaline. When adding CORT or MK571 to ADR plus GF120918, no significant differences were found in ADR content (Figure 3B). To verify whether the GF120918 inhibition of EMT was dose dependent, we incubated the cardiomyocytes with $500 \mu \mathrm{M}$ ADR with two other concen- trations of GF120918, 1 and $0.1 \mu \mathrm{M}(37,38)$. In this study, we showed that $10 \mu \mathrm{M}$ GF120918 decreased ADR within the cells to about $12 \%$ when compared to ADR group, while $1 \mu \mathrm{M}$ GF120918 resulted in a decrease to about 58\% (Figure 3C). Studies with $20 \mu \mathrm{M}$ GF120918 did not provide statistical differences in ADR intracellular concentration when compared to $10 \mu \mathrm{M}$ GF120918 (data not shown). No statistical difference was found between the ADR plus 0.1 $\mu$ M GF120918 group and the ADR group.

Intracellular ADR content in cardiomyocytes was also evaluated after incubation with quinidine and cyclosporin. Quinidine and cyclosporin had no significant effect in intracellular ADR (Figure 3D) and GSH adduct content (data not shown). No interfering chromatographic peaks were found in cyclosporin group, quinidine group, GF120918 group, or the vehicle group (data not shown).

Cellular Viability. ADR (maximum concentration of 500 $\mu \mathrm{M}$ ), GF120918, MK-571, DMSO (maximum concentration of $2 \%$ ), CORT, quinidine, and cyclosporin were not cytotoxic as verified using the LDH leakage test (data not shown). Verapamil in concentrations described to inhibit P-gp $(10 \mu \mathrm{M})(17,39)$ lead to decreased viability. As such, verapamil was not further used in our studies.

\section{Discussion}

In the present work, we started by characterizing EMT in freshly isolated cardiomyocytes, with a well-known EMT inhibitor, CORT (35). By using two very distinct ADR concentrations, 500 and $10 \mu \mathrm{M}$, we were able to demonstrate that these cells are a suitable model to investigate EMT and, therefore, its role in the oxidation process of catecholamines within the cells. Our previous work has shown the ability of ADR to undergo a complex oxidation process, leading to formation of a glutathionyl adduct [5-(glutathion-S-yl)adrenaline] (Figure 4) in the cardiomyocytes, still detectable with ADR concentrations as low as $25 \mu \mathrm{M}$ (5). In the present study, we found further evidence that GSH adduct is formed within the cells, as a marked decrease of intracellular 5-(glutathion- $S$ yl)adrenaline was observed when CORT blocked ADR uptake (Figure 1B). This depletion is directly associated to the decrease of ADR within the cardiomyocytes. CORT decreased ADR within the cells to about $13 \%$ when compared to ADR group (Figure 1A), exactly the same reduction observed in the adduct formation in ADR with CORT group. We excluded the inward transport of the adduct through EMT, since incubating with preformed 5-(glutathion-S-yl)adrenaline alone or with CORT lead to no differences in the values of the referred adduct in the cardiomyocytes.

The cytotoxicity of catechol-GSH adducts has been frequently reported $(6,10,14-16)$. As high values of 5-(glutathion- $S$ yl)adrenaline were found in the incubation medium (5), we assumed that a possible transporter for GSH conjugate efflux could be present in cardiomyocytes with a conceivable protective role (23). Thus, we decided to investigate the involvement of $\mathrm{ABC}$ transporters in the efflux of GSH conjugates and further elucidate the efflux transport mechanisms in freshly isolated cardiomyocytes. GSH is a chemically reactive tripeptide and, consequently, can form different types of adducts, namely, GSHthioethers, -thioesthers, and -mercaptides (23). GSH-conjugated metabolites can be exported from the cells, their efflux being often mediated by the MRP1 transporter. MRP1 is expressed at moderate levels in most normal tissues, except in the liver, where it is usually barely detectable $(24,26)$, conferring drug resistance to tumor cells and protecting cells from toxic insults 
A
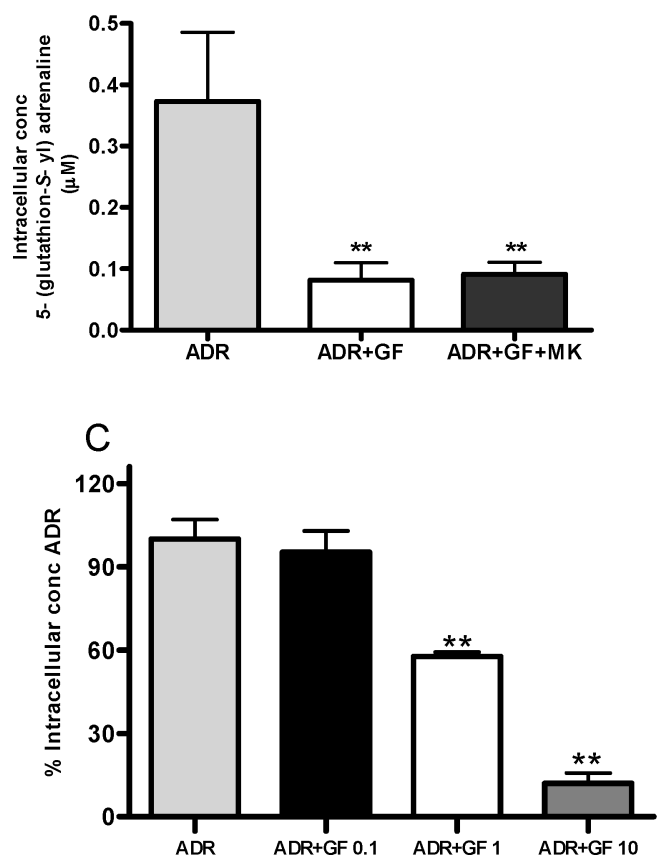

B

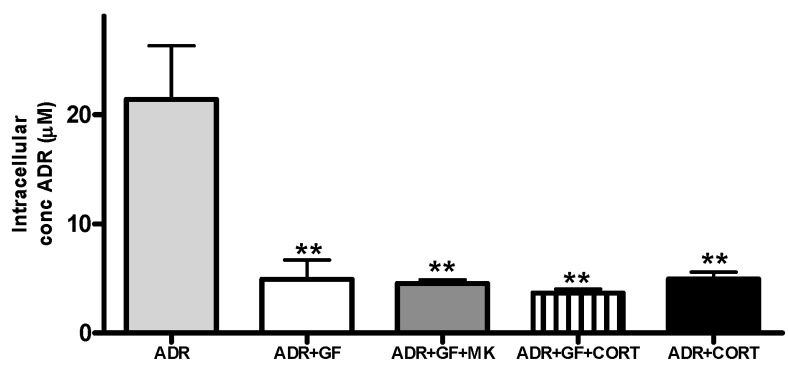

D

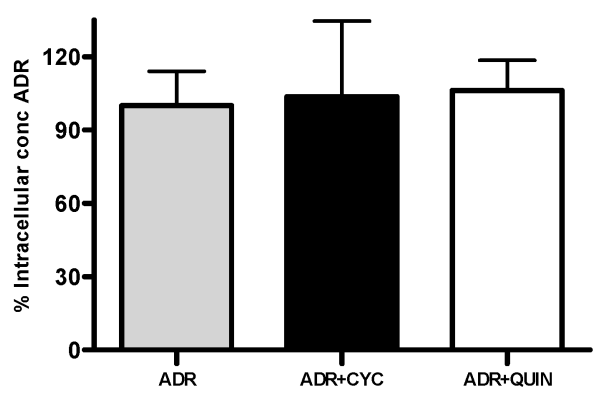

Figure 3. (A) Intracellular levels of 5-(glutathion-S-yl)adrenaline in cardiomyocytes exposed to $500 \mu \mathrm{M}$ ADR, $500 \mu \mathrm{M}$ ADR plus GF120918 (10 $\mu \mathrm{M}$ ), and $500 \mu \mathrm{M}$ ADR plus GF120918 plus MK-571 for $3 \mathrm{~h}$. Statistical comparisons were made using Kruskal-Wallis test, followed by the Student-Newman-Keuls post hoc test (**p $<0.01$ vs $500 \mu \mathrm{M}$ ADR) of six independent experiments. (B) Intracellular levels of ADR of myocytes exposed to $500 \mu \mathrm{M}$ ADR, $500 \mu \mathrm{M}$ ADR with GF120918 $(10 \mu \mathrm{M}), 500 \mu \mathrm{M}$ ADR with GF120918 and MK-571, $500 \mu \mathrm{M}$ ADR with GF120918 and CORT, and $500 \mu \mathrm{M}$ ADR with CORT for $3 \mathrm{~h}$. Statistical comparisons were made using Kruskal-Wallis test, followed by the Student - Newman - Keuls post hoc test (**p $<0.01$ vs $500 \mu \mathrm{M}$ ADR) of six independent experiments. (C) Intracellular levels of ADR in cardiomyocytes after the incubation with $500 \mu \mathrm{M}$ ADR and $500 \mu \mathrm{M}$ ADR with GF120918 $(0.1,1$, and $10 \mu \mathrm{M})$ at $3 \mathrm{~h}$. Results are presented as means \pm SD from six different experiments when compared to $500 \mu \mathrm{M}$ ADR, which was set to $100 \%$. Statistical comparisons were made using Kruskal-Wallis test, followed by the Student-Newman-Keuls post hoc test (**p $<0.01$ vs $500 \mu \mathrm{M}$ ADR). (D) Intracellular levels of ADR in myocytes exposed to $500 \mu \mathrm{M}$ ADR, $500 \mu \mathrm{M}$ ADR with cyclosporine (CYC) at $12 \mu \mathrm{M}$, and $500 \mu \mathrm{M}$ ADR with quinidine (QUIN) at $100 \mu \mathrm{M}$ for 3 h. Results are presented as means $\pm \mathrm{SD}$ from six different experiments when compared with $500 \mu \mathrm{M}$ ADR, which was set to $100 \%$.

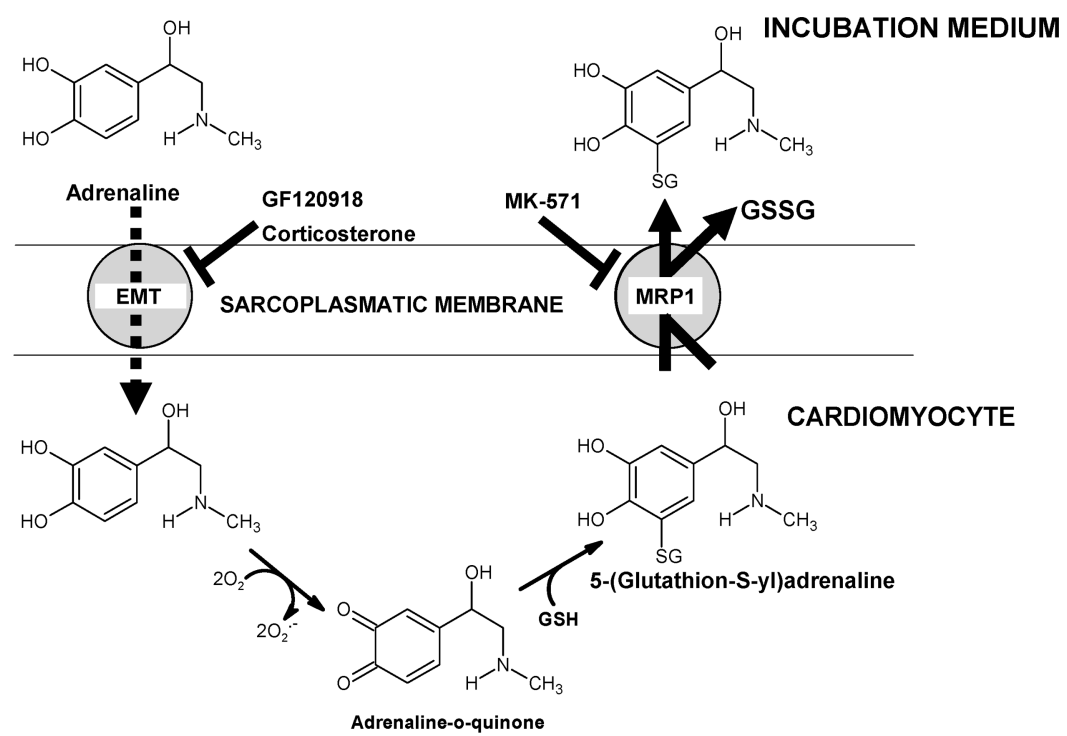

Figure 4. Characterization of transport mechanisms in freshly isolated cardiomyocytes. The oxidation process of ADR involves the formation of several reactive intermediaries and reactive species in cardiomyocytes. The oxidation process of ADR initially involves its conversion to $o$-quinone that can react with GSH to form the corresponding GSH conjugates or react with other nucleophilic groups in the cells, namely, proteins. The 5-(glutathion-S-yl)adrenaline and GSSG are transported out of the cells by multidrug resistant protein 1 (MRP1). MRP1 is inhibited by MK-571. EMT is active in these calcium-tolerant cardiomyocytes. EMT is blocked by CORT and GF120918, reported as a P-gp inhibitor. These cells after isolation and incubation seem to maintain the characteristics of adult heart cells, since they show EMT and MRP1 active transport but no signs of P-gp activity.

$(23,24)$. In the present study, we have shown that MRP1 has an important role in exporting 5-(glutathion-S-yl)adrenaline formed by ADR oxidation, although we do not exclude the involvement of other transporters, as well. When cardiomyocytes were exposed to ADR and MK-571, the intracellular GSH adduct (Figure 2A) and GSSG levels (and consequently GSHt) (Figure 2B,C) significantly increased. Previous evidence suggests that the decrease of GSH in the ADR group is related to 
the formation of reactive species (5), at the same time as the formation of those reactive species was not inhibited by exposition to adrenergic antagonists, prazosin, and propranolol (our unpublished results). The cardiomyocytes exposure to ADR increased intracellular GSSG, but cells protect themselves through MRP1 efflux. It is feasible that the transport of GSSG and 5-(glutathion- $S$-yl)adrenaline in the cardiomyocytes exposed to ADR occurs through the same MRP1 (26).

Additionally, we aimed to gather more data about other active transport mechanisms in freshly isolated cardiomyocytes, namely, P-gp. The controversy whether cardiomyocytes normally have functional P-gp is an actual subject. It is generally accepted that, in the normal heart, $\mathrm{P}$-gp is absent in cardiomyocytes, being only expressed in endothelial cells of capillaries and arterioles $(40-42)$. The fact remains that several works showed that P-gp inhibitors alter transport mechanisms in cardiomyocytes $(17,39,43)$. Some authors attribute this fallacious effect to the isolation or culture of the cells, which become able to express P-gp (44). We used a well-characterized P-gp substrate, quinidine (34), to determine whether P-gp activity was present in our freshly isolated cardiomyocytes. With that objective, we performed an efflux study, in which cells were incubated with the P-gp substrate to ensure its intracellular distribution, followed by the incubation with a P-gp inhibitor, $\operatorname{GF} 120918(37,38)$. The cells showed no signs of P-gp activity when exposed to quinidine, since its intracellular concentration was not altered by the P-gp inhibitor. This demonstrated that in what concerns this specific transporter, the isolated cardiomyocytes retained adult cardiac cells characteristics.

Another objective of this work was to evaluate the influence of various transport inhibitors in the formation and efflux of 5-(glutathion- $S$-yl)adrenaline. When incubating ADR with P-gp inhibitor GF120918, the 5-(glutathion-S-yl)adrenaline levels in cardiomyocytes decreased dramatically, even with simultaneous incubation with MK-571 (Figure 3A). With such an intriguing result, further investigation was required, through the measurement of intracellular ADR levels. Incubation of cardiomyocytes with GF120918 at $10 \mu \mathrm{M}$ resulted in an unexpected decrease in intracellular ADR levels (down to 12\%) (Figure 3B), thus explaining the compromised 5-(glutathion- $S$-yl)adrenaline synthesis. This ADR decrease was shown to be GF120918 concentration dependent. Noteworthy, even using a 500 times higher concentration of ADR $(500 \mu \mathrm{M})$, the GF120918 $(1 \mu \mathrm{M})$ had a significant inhibition effect upon ADR uptake mechanisms (Figure 3C).

Using the same concentrations of ADR (500 $\mu \mathrm{M})$, GF120918 was shown to be even more potent than the specific EMT inhibitor, CORT. In fact, with a 10 times lower concentration (10 $\mu \mathrm{M})$, GF120918 decreased ADR within the cells to about the same amount as CORT at $100 \mu \mathrm{M}$ (Figure 3B). Even when combining CORT $(100 \mu \mathrm{M})$ and GF120918 $(10 \mu \mathrm{M})$, intracellular ADR is still detected (Figure 3B). This may reveal the presence of an additional ADR uptake mechanism in this model.

A P-gp inhibitor (cyclosporin) and a P-gp substrate (quinidine) were used to test their influence in ADR uptake in the isolated cardiomyocytes. Cyclosporin ( 3 and $12 \mu \mathrm{M}$ ) had no effect in ADR concentrations within the cardiomyocytes (Figure 3D). Other transporters could be involved in the uptake of ADR in cardiomyocytes, like PMAT (32). Quinidine is known to be a P-gp subtstrate and also PMAT and OCT1 and -2 inhibitor (31). In this experimental model, quinidine did not interfere with ADR uptake. In the present concentrations and model, EMT seems to have higher relevance in ADR transport. However, and as ADR uptake was not fully inhibited, even by $20 \mu \mathrm{M}$ GF120918 or by $10 \mu \mathrm{M}$ GF120918 plus $100 \mu \mathrm{M}$ CORT, it is conceivable that other import mechanisms may be involved.

The present work has the merit of showing that caution must be taken when using "specific" inhibitors that, in this particular case, even at micromolar concentrations, inhibit other transporters, namely, EMT. A decrease in EMT may lead to an accumulation of catecholamines in the intersticial space with possible toxic effects. The catecholamine transporters function as part of the uptake and metabolizing systems primarily responsible for inactivation of the transmitters, as the enzymes involved in the inactivation of catecholamines are located intracellularly (27). EMT impairment can lead to an increase of catecholamine levels, leading to sustained sympathicomimetic activation, which may affect many organs, inclusively the heart with inauspicious toxic results $(30,45,46)$. Moreover, the inhibition of EMT has a higher influence in the inactivation of ADR, since EMT favors ADR over noradrenaline or dopamine (30). Notwithstanding, Yang and co-workers had shown that CORT, a well-characterized uptake ${ }_{2}$ inhibitor, is a P-gp inhibitor (47); the inverse relation with a P-gp inhibitor was never considered until know, to the best of our knowledge.

Our short-term studies require higher concentrations of catecholamines than the ones expected in vivo (46). To study the transport at physiological concentrations, most of the transport studies in isolated cells are performed with radiochemical analysis $(35,48)$, with the inherent problems related to the storage, handling, and waste disposal of radioactive materials. Even so, we showed that these isolated cardiomyocytes perform well. Therefore, they are a reliable model for studying ADR and its metabolite transport.

In conclusion, EMT is active in freshly isolated rat cardiomyocytes, thus incorporating ADR, which undergoes subsequent intracellular oxidation reactions (Figure 4). The oxidation process of $\mathrm{ADR}$ involves the formation of many reactive intermediaries and reactive species in cardiomyocytes, namely, GSH adduct and GSSG. The 5-(glutathion- $S$-yl)adrenaline and GSSG efflux in cardiomyocytes are carried out by MRP1 (Figure 4).

Acknowledgment. This work received financial support from the Portuguese State through "Fundação para a Ciência e Tecnologia" (FCT) (project PPCDT/SAU-OBS/55849/2004). V.M.C. acknowledges FCT for her Ph.D. grant (SFRD/BD/ 17677/ 2004) and GlaxoSmithKline for providing the GF120918.

\section{References}

(1) Remião, F., Carvalho, M., Carmo, H., Carvalho, F., and Bastos, M. L. (2002) $\mathrm{Cu}^{2+}$-induced isoproterenol oxidation into isoprenochrome in adult rat calcium-tolerant cardiomyocytes. Chem. Res. Toxicol. 15, 861-869.

(2) Bindoli, A., Rigobello, M. P., and Deeble, D. J. (1992) Biochemical and toxicological properties of the oxidation products of catecholamines. Free Radical Biol. Med. 13, 391-405.

(3) Dhalla, S. N., Sasaki, H., Mochizuki, S., Dhalla, S. K., Liu, X., and Elimban, V. (2001) Catecholamine-induced cardiomyopathy. In Cardiovascular Toxicology (Acosta, D. J., Ed.) pp 269-318, Taylor and Francis, London.

(4) Flaherty, J. T. (1991) Myocardial injury mediated by oxygen free radical. Am. J. Med. 91, 79S-88S.

(5) Costa, V. M., Silva, R., Ferreira, L. M., Branco, P. S., Carvalho, F., Bastos, M. L., Carvalho, R. A., Carvalho, M., and Remião, F. (2007) Oxidation process of adrenaline in freshly isolated rat cardiomyocytes: Formation of adrenochrome, quinoproteins and GSH-adduct. Chem. Res. Toxicol. 20, 1183-1191.

(6) Carvalho, M., Remião, F., Milhazes, N., Borges, F., Fernandes, E., Monteiro, M. C., Gonçalves, M. J., Seabra, V., Amado, F., Carvalho, 
F., and Bastos, M. L. (2004) Metabolism is required for the expression of ecstasy-induced cardiotoxicity in vitro. Chem. Res. Toxicol. 17, 623632.

(7) Remião, F., Carmo, H., Carvalho, F., and Bastos, M. L. (2001) Copper enhances isoproterenol toxicity in isolated rat cardiomyocytes. Cardiovasc. Toxicol. 1, 195-204.

(8) Remião, F., Rettori, D., Han, D., Carvalho, F., Bastos, M. L., and Cadenas, E. (2004) Leucoisoprenochrome-o-semiquinone formation in freshly isolated adult rat cardiomyocytes. Chem. Res. Toxicol. 17, $1584-1590$

(9) Monks, T. J., and Lau, S. S. (1992) Toxicology of quinone-thioethers. Crit. Rev. Toxicol. 22, 243-270.

(10) Monks, T. J., and Lau, S. S. (1997) Biological reactivity of polyphenolic-glutathione conjugates. Chem. Res. Toxicol. 10, 1296-1313.

(11) Spencer, J. P. E., Jenner, P., Daniel, S. E., Lees, A. J., Marsden, D. C., and Halliwell, B. (1998) Conjugates of catecholamines with cysteine and GSH in Parkinson's disease: Possible mechanism of formation involving reactive oxygen species. J. Neurochem. 71, 2112-2122.

(12) Spencer, J. P. E., Whiteman, M., Jenner, P., and Halliwel, B. (2002) 5-S-Cysteinyl-conjugates of catecholamines induce cell damage, extensive DNA base modication and increases in caspase- 3 activity in neurons. J. Neurochem. 81, 122-129.

(13) Carvalho, M., Hawksworth, G., Milhazes, N., Borges, F., Monks, T. J., Fernandes, E., Carvalho, F., and Bastos, M. L. (2002) Role of metabolites in MDMA (ecstasy)-induced nephrotoxicity: An in vitro study using rat and human renal proximal tubular cells. Arch. Toxicol. 76, 581-588.

(14) Capela, J. P., Meisel, A., Abreu, A. R., Branco, P. S., Ferreira, L. M., Lobo, A. M., Remião, F., Bastos, M. L., and Carvalho, F. (2006) Neurotoxicity of ecstasy metabolites in rat cortical neurons and influence of hyperthermia. J. Pharmacol. Exp. Ther. 316, 53-61.

(15) Capela, J. P., Macedo, C., Branco, P. S., Ferreira, L. M., Lobo, A., Fernandes, E., Remião, F., Bastos, M. L., Dirnagl, U., Meisel, A., and Carvalho, F. (2007) Neurotoxicity mechanisms of thiother ecstasy metabolites. Neuroscience 146, 1743-1757.

(16) LaVoie, M. J., and Hastings, T. G. (1999) Dopamine quinone formation and protein modification associated with the striatal neurotoxicity of methamphetamine: evidence against a role for extracellular dopamine. J. Neurosci. 19, 1484-1491.

(17) Ghosh, S., Ting, S., Lau, H., Pulinilkunnil, T., An, D., Qi, D., Abrahani, M. A., and Rodrigues, B. (2004) Increased efflux of glutathione conjugate in acutely diabetic cardiomyocytes. Can. J. Physiol. Pharmacol. 82, 879-887.

(18) Juliano, R. L., and Ling, V. (1976) A surface glycoprotein modulating drug permeability in Chinese hamster ovary cell mutants. Biochim. Biophys. Acta 455, 152-162.

(19) Cole, S. P., Bhardwaj, G., Gerlach, J. H., Mackie, J. E., Grant, C. E., Almquist, K. C., Stewart, A. J., Kurz, E. U., Duncan, A. M., and Deeley, R. G. (1992) Overexpression of a transporter gene in a multidrug resistant human lung cancer cell line. Science 258, 16501654.

(20) Allikmets, R., Schriml, L. M., Hutchinson, A., Romano-Spica, V., and Dean, M. (1998) A human placenta-specific ATP-binding cassette gene $(\mathrm{ABCP})$ on chromosome $4 \mathrm{q} 22$ that is involved in multidrug resistance. Cancer Res. 58, 5337-5339.

(21) Borst, P., and Elferink, O. R. (2002) Mammalian ABC transporters in health and disease. Annu. Rev. Biochem. 71, 537-592.

(22) Schinkel, A. H., and Jonker, J. W. (2003) Mammalian drug efflux transporters of the ATP binding cassette (ABC) family: An overview. Adv. Drug Delivery Rev. 55, 3-29.

(23) Ballatori, N., Hammond, C. L., Cunningham, J. B., Krance, S. M., and Marchan, R. (2005) Molecular mechanisms of reduced glutathione transport: role of the MRP/CFTR/ABCC and OATP/SLC21A families of membrane proteins. Toxicol. Appl. Pharmacol. 204, 238-255.

(24) Leslie, E. M., Deeley, R. G., and Cole, S. P. (2007) Multidrug resistance proteins: Role of P-glycoprotein, MRP1, MRP2 and BCRP (ABCG2) in tissue defense. Toxicol. Appl. Pharmacol. 204, 216-227.

(25) McDevitt, C. A., and Callaghan, R. (2007) How can we best structure information on P-glycoprotein to design inhibitors? Pharmacol. Ther. 113, 429-441.

(26) Cole, S. P. C., and Deeley, R. G. (2006) Transport of glutathione and glutathione conjugates by MRP1. Trends Pharmacol. Sci. 27, 438446.

(27) Eisenhofer, G. (2001) The role of neuronal and extraneuronal plasma membrane transporters in the inactivation of peripheral catecholamines. Pharmacol. Ther. 91, 35-62.

(28) Iversen, L. L. (1965) The uptake of catecholamines at high perfusion concentrations in the rat isolated heart: A novel catecholamine uptake process. Br. J. Pharmacol. 24, 18-33.
(29) Malmfors, T. (1967) Fluorescence histochemical studies on the uptake, storage, and release of catecholarnines. Circ. Res. $X X-X X I, 11125-$ 11142 .

(30) Westfall, T. C., and Westfall, D. P. (2006) Adrenergic agonists and antagonists. In Goodman \& Gilman's The Pharmacological Basis of Therapeutics (Brunton, L. L., Lazo, J. S., and Parker, K. L., Eds.) McGraw-Hill, New York.

(31) Engel, K., and Wang, J. (2005) Interaction of organic cations with a newly identified plasma membrane monoamine transporter. Mol. Pharmacol. 68, 1397-1407.

(32) Engel, K., Zhou, M., and Wang, J. (2004) Identification and characterization of a novel monoamine transporter in the human brain. J. Biol. Chem. 279, 50042-50049.

(33) Silva, R., Boldt, S., Costa, V. M., Carmo, H., Carvalho, M., Carvalho, F., Bastos, M. L., Lemos-Amado, F., and Remião, F. (2007) Evaluation of GSH adducts of adrenaline in biological samples. Biomed. Chromatogr. 21, 670-679.

(34) Varma, M. V. S., Sarkar, M., Kapoor, N., and Panchagnula, R. (2005) $\mathrm{pH}$-dependents functional activity of P-glycoprotein in limiting intestinal absorption of protic drugs 1. Simultaneous determination of quinidine and markers in rat in situ perfusion samples. J. Chromatogr. B 816, 243-249.

(35) Obst, O., Rose, H., and Kammermeier, H. (1996) Characterization of catecholamine uptake, in isolated cardiac myocytes. Mol. Cell. Biochem. 163-164, 181-183.

(36) Carvalho, M., Milhazes, N., Remião, F., Borges, F., Fernandes, E., Amado, F., Monks, T. J., Carvalho, F., and Bastos, M. L. (2004) Hepatotoxicity of 3,4-methylenedioxyamphetamine and alpha-methyldopamine in isolated rat hepatocytes: formation of glutathione conjugates. Arch. Toxicol. 78, 16-24.

(37) Declèves, X., Niel, E., Debray, M., and Scherrmann, J.-M. (2006) Is P-glycoprotein (ABCB1) a phase 0 or a phase 3 colchicine transporter depending on colchicine exposure conditions? Toxicol. Appl. Pharmacol. 217, 153-160.

(38) Schutte, M. E., Freidig, A. P., van de Sandt, J. J. M., Alink, G. M., Rietjens, I. M. C. M., and Groten, J. P. (2006) An in vitro and in silico study on the flavonoid-mediated modulation of the transport of 2-amino-1-methyl-6-phenylimidazo[4,5-b]pyridine (PhIP) through Caco-2 monolayers. Toxicol. Appl. Pharmacol. 217, 204-215.

(39) Gruber, A., Larsson, R., Nygren, P., Björkholm, M., and Peterson, C. (1994) A non-P-glycoprotein-mediated mechanism of vincristine transport which is affected by resistance modifiers and present in chemosensitive cells. Leukemia 8, 985-989.

(40) Meissner, K., Sperker, B., Karsten, C., zu Schwabedissen, H. M., Seeland, U., Bohm, M., Bien, S., Dazert, P., Kunert-Keil, C., Vogelgesang, S., Warzok, R., Siegmund, W., Cascorbi, I., Wendt, M., and Kroemer, H. K. (2002) Expression and localization of Pglycoprotein in human heart: effects of cardiomyopathy. J. Histochem. Cytochem. 50, 1351-1356.

(41) Lazarowski, A. J., Garcia Rivello, H. J., Vera Janavel, G. L., Cuniberti, L. A., Cabeza Meckert, P. M., Yannarelli, G. G., Mele, A., Crottogini, A. J., and Laguens, R. P. (2005) Cardiomyocytes of chronically ischemic pig hearts express the MDR-1 gene-encoded P-glycoprotein. J. Histochem. Cytochem. 53, 845-850.

(42) Laguens, R. P., Lazarowski, A. J., Cuniberti, L. A., Vera Janavel, G. L., Cabeza Meckert, P. M., Yannarelli, G. G., del Valle, H. F., Lascano, E. C., Negroni, J. A., and Crottogini, A. J. (2007) Expression of the MDR-1 gene-encoded P-glycoprotein in cardiomyocytes of conscious sheep undergoing acute myocardial ischemia followed by reperfusion. J. Histochem. Cytochem. 55, 191-197.

(43) Estevez, M. D., Wolf, A., and Schramm, U. (2000) Effect of PSC 833 , verapamil and amiodarone on adriamycin toxicity in cultured rat cardiomyocytes. Toxicol in Vitro 14, 17-23.

(44) Sukhai, M., and Piquette-Miller, M. (2000) Regulation of the multidrug resistance genes by stress signals. Pharm. Pharm. Sci. 3, 268-280.

(45) Goldstein, D. S. (2003) Catecholamines and stress. Endocr. Regul. 37, 69-80.

(46) Goldstein, D. S., Eisenhofer, G., and Kopin, I. J. (2003) Sources and significance of plasma levels of catechols and their metabolites in humans. J. Pharmacol. Exp. Ther. 305, 800-811.

(47) Yang, C. P., DePinho, S. G., Greenberger, L. M., Arceci, R. J., and Horwitz, S. B. (1989) Progesterone interacts with P-glycoprotein in multidrug-resistant cells and in the endometrium of gravid uterus. J. Biol. Chem. 264, 782-788.

(48) Shirasaka, Y., Sakane, T., and Yamashita, S. (2006) Effect of P-glycoprotein expression levels on the concentration-dependent permeability of drugs to the cell membrane. J. Pharm. Sci. 97, 553-565.

\section{TX8002577}

